\title{
The application of PTFE coating on spherical joints
}

\section{JieZhang $^{\mathrm{a}}$, DanWang ${ }^{\mathrm{b}}$, WuyiChen ${ }^{\mathrm{c}}$}

School of Mechanical Engineering and Automation, Beihang University, Beijing 100191, China

amercury1307217@126.com, ${ }^{\mathrm{b} C}$ Corresponding

author:wd_smea@buaa.edu.cn, 'wychen@buaa.edu.cn

\begin{abstract}
Keywords: Solid lubricants, PTFE coating, Spherical joint, Parallel Mechanism
\end{abstract}
\begin{abstract}
Spherical joints are wildly used in parallel mechanisms. This paper applied polytetrafluoroethylene (PTFE) on the surface of spherical joints, aiming at reducing both frictional resistance and clearance of this kind of joints. Firstly, three kinds of solid lubricants were coated on flat samples and their friction performances were investigated. Then the best of the three was used as friction-reducing layer filling the gap between the ball and the support in a joint. Finally, periodic rotating abrasion test was conducted on this coated spherical joint. Experimental results showed that PTFE coating could effectively reduce the clearance of ball joints and prevent components' surfaces from rapid wear, without increasing friction significantly.
\end{abstract}

\section{Introduction}

Spherical joints are the most commonly used type of joints in parallel manipulators, but friction force and clearance in this kind of joints considerably affect the functional performance of mechanism. On the other hand, reducing the clearance in a spherical joint will increase the friction resistance between the contact surfaces, which is also a negative factor in joint performance. Conciliating the conflict between friction and clearance in spherical joints could help improve the property of this kind of joints.

Kinematic accuracy of mechanisms with joints is highly relevant to joint clearances. Reference [1] established contact model of joints with clearance and then applied this joint model into a four-bar linkage model, and comparisonoflinkage kinematic behavior under different clearances had been conducted. Reference [2] took the same method to study a 3-RRR parallel mechanism, and their results indicated that joint clearance had considerably impact on system stability and precision.

Reducing friction force in joints is another meaningful research object. Different lubricating methods have been adopted and studies on friction have been conducted. [3- 5] In a spherical joint, both load and frictional coefficient were directly proportional to friction, so a smaller frictional coefficient between contact-pair could contribute to lower friction resistance. PTFE has very low friction coefficient, and has been widely used in mechanical fields.[6-8] Test methods on materials' tribology properties are well-developed, and industrial coatings with required properties for specific application can be easily obtained.

In this paper, three solid lubricant coatings had been tested under ball-on-flat contact, and their friction and wear performance had been compared. Then, one coating with the best tribology properties was applied on the contact surfaces in spherical joint, aiming at keeping low friction while reducing the clearance of joints.

\section{Design of spherical joints with coating}

A typical spherical joint consists of a ball, a cover and a base, Fig.1. The contact surfaces of the ball and the base/cover are prone to wear due to reciprocating sliding friction. With the increase of wear, the clearance in joint increases and the accuracy decreases. For this reason, both mechanical performance and wear resistance need to be taken into account when selecting friction pair in a spherical joint. Friction pair composed of metallic and non-metallic materials shows higher resistance to adhesive wear, and using metal as substrate and non-metal as a coating layer proposes 
a good solution.

Pre-reserved gap isa critical dimension in the design of spherical joints. Enlarging the gap means the loss of accuracy while narrowing the gap means the increase of friction. Using the empirical design method, the clearance of spherical joint should be less than $10 \mu \mathrm{m}$ after precision finishing.

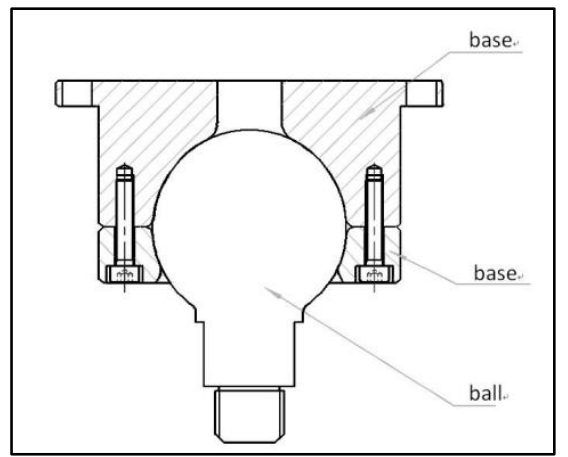

Fig. 1Aschematic diagram for a spherical joint

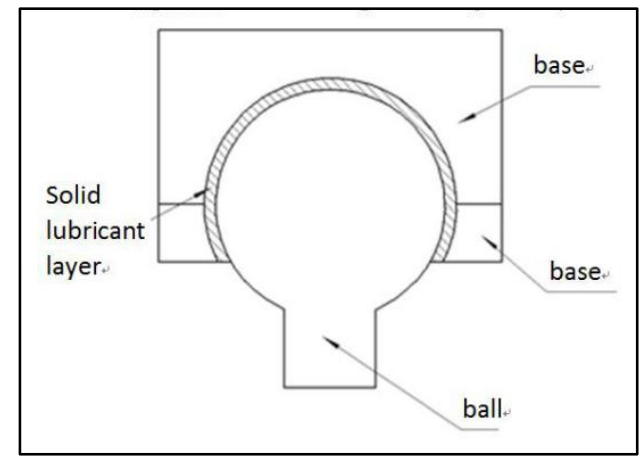

Fig.2 A schematic diagram for a coated joint

In the design of coated spherical joints, the thickness of the coating on the ball needs to be slightly larger than the reserved clearance of the joint at the pre-design stage. This design allows the gap to be completely filled up by compressing lubricant coating, Fig. 2. While the low friction coefficient of coating guarantees the smooth sliding between the ball and the base/cover. Both components of spherical joint are prevented from rapid wear.

\section{Coating test and analysis}

\subsection{Specimen preparation.}

Two kinds of PTFE coatings were chosen from Teflon Industrial coatings, and the MoS2 coating was provided by Lanzhou Institute of Chemical Physics. Table 1 showed the specification and properties of these coatings and specimens. The upper specimen was a sphere made of Steel 40Cr, the same material with spherical joint used in follow-on test. The substrate material is Steel 40Cr, and its top and bottom surfaces both got polished before coating and curing.

Table 1 Specification and properties of coatings and specimens

\begin{tabular}{cccccc}
\hline \multirow{4}{*}{ Coatings } & Material & $\begin{array}{c}\text { Thickness of } \\
\text { Coating } \delta(\mu \mathrm{m})\end{array}$ & Size $(\mathrm{mm})$ & $\begin{array}{c}\text { Density } \\
\rho\left(\mathrm{g}^{*} \mathrm{~cm}^{-3}\right)\end{array}$ & $\begin{array}{c}\text { Surface } \\
\text { roughness } \\
\text { Ra }(\mu \mathrm{m})\end{array}$ \\
\hline & $\begin{array}{c}\text { Teflon } \\
\text { PTFE958303 }\end{array}$ & $16 \pm 2$ & - & 1.04 & 1.71 \\
\cline { 2 - 6 } & $\begin{array}{c}\text { Teflon } \\
\text { PTFE959203 }\end{array}$ & $16 \pm 2$ & - & 1.02 & 1.78 \\
\cline { 2 - 6 } $\mathrm{MoS}_{2}$ HM-1100A & $20 \pm 2$ & - & 3.8 & 1.62 \\
\hline Upper specimen & $40 \mathrm{Cr}$ & - & $\mathrm{S} \Phi 6.3$ & 7.85 & 0.8 \\
\hline $\begin{array}{c}\text { Substrate } \\
\text { material }\end{array}$ & $40 \mathrm{Cr}$ & - & $20 \times 25 \times 3$ & 7.85 & 0.8 \\
\hline
\end{tabular}

Substrate specimen was firstly sanded with 120-grit Al2O3, and then cleaned with acetone and dried. The coatings were sprayed onto the top surfaces of specimens with airbrush. Then, the coatings were cured at $150 \pm 5^{\circ} \mathrm{C}$ and $280 \pm 5^{\circ} \mathrm{C}$ for one hour respectively, and air cooled to room temperature.

\subsection{Frictional Test.}

Frictional test was performed on tribology tester (Model: Bruker-UMT2). Experiment parameters, such as load and sliding speed, could be set as required, and all four materials (including three kinds of coatings and substrate metal) were tested under three sliding velocity levels and five load levels. Table 2 listed out these parameters used in the test. The frictional force and wear depth was recorded in the experimental process with software, and the frictional 
coefficients could be calculated accordingly.

Table 2Friction test conditions

\begin{tabular}{|c|c|}
\hline Parameter & Value \\
\hline Load $\quad F(N)$ & 20/40/60/80/100 \\
\hline Sliding velocity $v(\mathrm{~mm} \cdot \mathrm{s}-1)$ & $0.5 / 5 / 50$ \\
\hline Environment temperature T ( & 25 \\
\hline Running time $\mathrm{t}(\mathrm{min})$ & 5 \\
\hline
\end{tabular}

\subsection{Results and discussion.}

Frictional properties of three coatings were compared in Fig.3 and Fig. 4. Fig. 3 showed the dynamic friction coefficients of four materials under a velocity of $5 \mathrm{~mm} / \mathrm{s}$, and the comparison of frictional properties between metal and coatings could also be seen. Fig. 4 showed the dynamic friction coefficients of three coatings under two speed-levels, i.e., $0.5 \mathrm{~mm} / \mathrm{s}$ and $50 \mathrm{~mm} / \mathrm{s}$, both used as selection references.Wear depths of different coatings were showed in Table3.

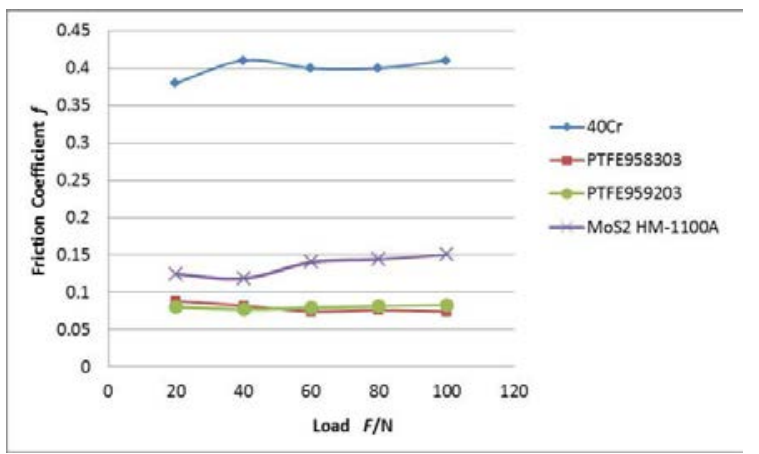

Fig.3 Friction coefficient of four materials varied with load

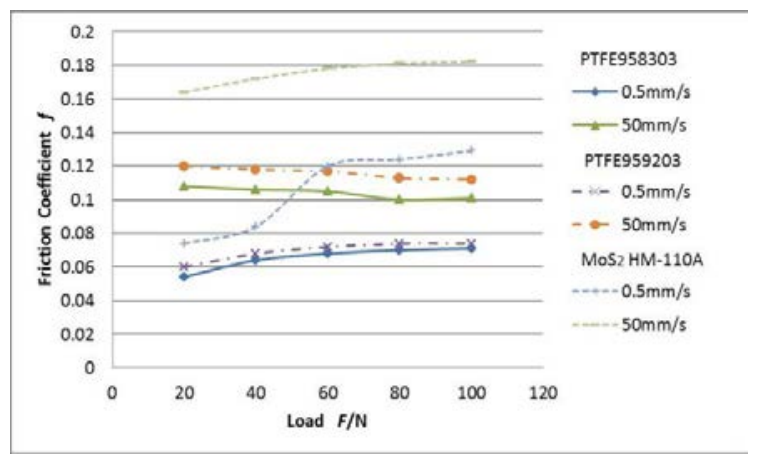

Fig.4Friction coefficient of three coatings varied with load at different speed-levels

Table 3Wear depth of three coating varied with load, at a sliding velocity of $5 \mathrm{~mm} / \mathrm{s}$

\begin{tabular}{ccccccc}
\hline \multirow{2}{*}{ Coatings } & Initial & \multicolumn{4}{c}{ Load F (N) } \\
\cline { 3 - 7 } & thickness & 20 & 40 & 60 & 80 & 100 \\
\hline MoS $_{2}$ HM-1100A & 1.62 & 12 & 14 & 14 & 18 & 18 \\
PTFE958303 & 1.71 & 10 & 10 & 8 & 10 & 12 \\
PTFE959203 & 1.78 & 12 & 10 & 14 & 12 & 12 \\
\hline
\end{tabular}

Combining figures and the data in Table above,some relations could be listed as follow:

a. During dry friction, three coating materials showed much lower friction coefficients than that of substrate metal. And coefficients gotten from tests were consisted with empirical values.

b. Two PTFE coatings possessed very close frictional property. Their friction coefficients tended to stabilize to a certain value with the increase of load. At low speed level, the value was positively correlated with load; at relatively high speed level, the value was negatively correlated with load. With three speed levels in the test, the same material's friction coefficient rose with the increase of velocity. This property indicated that the friction was not directly proportional to the load on the coating, which proposed a possibility to imply pre-tightening force on spherical joints while keeping friction at a low level. However, the decrease of frictional coefficient is too slight to significantly reduce the friction obviously.

c. The coefficient of MoS2 coating is around 0.5 times larger than that of PTFE coatings and it increased with the load at both high and low sliding speeds. When the load rose from $40 \mathrm{~N}$ to $60 \mathrm{~N}$ under the speed of $0.5 \mathrm{~mm} / \mathrm{s}$, the coefficient of friction yielded $50 \%$ increase. This result indicated that MoS2 was not an ideal coating for the spherical joints.

d. MoS2 coating possessed larger wear depth than PTFE coatings and was damaged more easily.Two PTFE coatings, on the other hand, held alike anti-wear properties. However, specimen with Teflon PTFE959203 appeared sporadic peeling, which indicated acute adhesive wear.

Therefore, Coating Teflon PTFE958303 was selected as the material applied on spherical ball in 
further experiment.

\section{Joint test and analysis}

\subsection{Experiments.}

Basic dimension for the ball joint used in the experiment was $\Phi 50 \mathrm{~mm}$. Before being coated, the ball and the base were ground to ensure the clearance of less than $15 \mu \mathrm{m}$ between these two parts, while movement of the ball was smooth enough.

Using the same approach with flat sample preparation, the ball of the joint was coated with a PTFE layer which thickness was from $15 \mu \mathrm{m}$ to $20 \mu \mathrm{m}$.

Driven by motor, the ball of the joint rotated at a uniform speed of $50 \mathrm{r} / \mathrm{min}$. A 2-min test was used as an observation unit to discuss the wear pattern on coating layer, and 15 groups of tests were conducted to assess the endurance of coating.

\subsection{Results and discussion.}

After 15 groups of rotating tests, phenomena observed in different phases were listed in time order in Table 4. Debris generated during the tests was collected and observed with a microscope, showed in Fig.5.

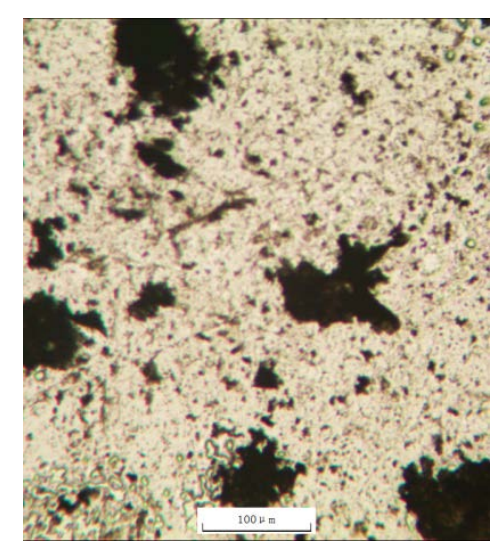

Fig. 5Debris of coating collected after joint tests(x100)

Table 4 Phenomena observed in rotating tests

Phases

Observation

\begin{tabular}{ll}
\hline Test 1 & There are considerable tiny black particles attached to contact surfaces. \\
Test 3-4 & $\begin{array}{l}\text { The number of black particles gains significantly with testing times; Worn zone on coating can } \\
\text { be observed from its color difference from unworn areas. }\end{array}$ \\
Test 5-10 & $\begin{array}{l}\text { Amount of debris collected gradually decrease. } \\
\text { Test11-15 }\end{array}$ \\
\hline
\end{tabular}

Phenomenamentioned above could be explained as:

a. During the first five tests, the coating went through initial stage of wear, and debris of coating accumulated quickly, adhering to contact surfaces and functioning as solid lubricant.

b. As the rotating tests went on, ragged surface of coating turned to be smoother, and wear gradually step into normal wear stage. Clearance of joint kept stable with self-adaption in initial wear stage.

c. No substrate metal was exposed indicates that the ball was still under the cover of coating. With the existing of coating, the clearance of the joint was limited in a value given in pre-design, and no rapid increase was detected.

Worn zone observedon coating hinted the wear-concentrated area on the ball when joint is in service. These areas should get extra attention in the study of clearance measurement. 


\section{Summary}

a. Solid lubricants could play roles as anti-friction layers applied on the contact surfaces in spherical joints and reduce joint's clearance. Their properties of low friction coefficients could keep the sliding friction in a low level while filling the gap between the ball and the base.

b. Both anti-friction and anti-wear qualities needed to be tested in the selection of coatings. PTFE coatings could provide a good lubricating service, and one kind of PTFE coating was used in the joint test, providing the viability of coated-joint design.

c. The surface roughness of coatings could be significantly reduced after a period of running-in, and the coating thickness on the ball could be self-adapted in initial wear stage. Debris of PTFE coating served as lubricant and prevented parts from rapid wear. As a result, the clearance and movement of the joint could keep stable for some time.

d. Theoretically, coating on the ball could greatly reduce the sliding friction in the joint. However, the effectiveness of it should be measured and analyzed in further comparative experiments.

\section{Acknowledgments}

This work is supported by the National Natural Science Foundation of China (Grant No. 51305013). The authors greatly thank for the support.

\section{References}

[1]. Zhang X C, Zhang X M, Chen Z. Dynamic analysis of a 3-RRR parallel mechanism with multiple clearance joints.Mechanism and Machine Theory.Vol. 78(2014), p.105-115.

[2]. Erkaya S, İbrahim Uzmay. Modeling and simulation of joint clearance effects on mechanisms having rigid and flexible links. Journal of Mechanical Science \& Technology.Vol. 28(2014) No.8, p. 2979-2986.

[3]. Faraz A, Payandeh S. Towards approximate models of Coulomb frictional moments in: (I) revolute pin joints and (II) spherical-socket ball joints. Journal of Engineering Mathematics,Vol. 40(2001) No.3, p. 283-296.

[4]. Smirnov Y P. Some frictional effects in a spherical joint. Soviet Applied Mechanics, Vol.17(1981) No. 10, p. 911-915.

[5]. Shin Y J. An analytical solution for spherical joint mechanism including Coulomb friction. Int. Pacific Conf. Autom. Eng., 1991, p. 383-389.

[6]. ShangguanQ Q, Chen X H. Tribological properties of lanthanum treated carbon fibers reinforced PTFE composite under dry sliding condition. Wear. Vol. 262(2007), p. 1419-1425.

[7]. Wang Y X, Yan F Y. A study on tribological behaviour of transfer films of PTFE/bronze composites.Wear. Vol. 262(2006), p. 876-882.

[8]. Sun L H, Yang N. Mechanical and tribological properties of polyoxymethylene modified with nanoparticles and solid lubricants. Polymer Engineering and Science, Vol. 48(2008), p. 1824-1832. 